\title{
A integração do cliente no processo de desenvolvimento de produto: revisão bibliográfica sistemática e temas para pesquisa
}

\author{
Mario Orestes Aguirre González ${ }^{\mathrm{a} *}$, José Carlos de Toledo ${ }^{\mathrm{b}}$ \\ a*mario@ct.ufrn.br, UFRN, Brasil \\ btoledo@dep.ufscar.br, UFSCar, Brasil
}

\begin{abstract}
Resumo
Este artigo apresenta uma revisão bibliográfica sistemática e aponta direções de pesquisa para o tema integração do cliente (IC) no processo de desenvolvimento de produto. Esse tema é considerado relevante na bibliografia das áreas de marketing, qualidade, inovação tecnológica e estratégia competitiva por permitir às empresas conhecerem melhor as reais necessidades do mercado, contribuir para o desenvolvimento de produtos mais adequados ao mercado e usar o conhecimento acumulado pelos clientes. Foram levantados 72 artigos que abordam esse tema no período de 1980 a 2008. 0 interesse pelo tema, no meio acadêmico, tem aumentado significativamente desde 2002, o que se constata pelo aumento no número de artigos publicados e pela incorporação do tema a capítulos de livros sobre gestão de desenvolvimento de produto. Identificaram-se as seguintes dimensões que compreendem o tema e que predominam nas pesquisas relatadas nos artigos analisados: fundamentos para a integração do cliente no processo de desenvolvimento do produto, critérios para seleção de clientes, tipos de interação entre a empresa e seus clientes e benefícios obtidos com a integração do cliente (IC). Para futuras pesquisas, recomenda-se aprofundar, por meio de estudo de casos e de survey, a compreensão que as empresas têm sobre o tema, as práticas adotadas e as relações e sinergia entre as dimensões, bem como as relações de causa e efeito entre as práticas e o desempenho do processo de desenvolvimento de produto.
\end{abstract}

Palavras-chave

Desenvolvimento de produto. Integração do cliente. Revisão bibliográfica sistemática. Integração do cliente no desenvolvimento de produto.

\section{Introdução}

Os modelos de sistemas de gestão da qualidade ISO 9000, de excelência em gestão de negócios (os chamados modelos dos Prêmios Nacionais da Qualidade) e os modelos de gestão da qualidade total enfatizam a satisfação e a lealdade dos clientes, e sua gestão, como fatores críticos para o sucesso e sustentabilidade dos negócios.

As empresas que adotam tais modelos realizam avaliações periódicas para acompanhar seu desempenho no índice satisfação dos clientes. Entretanto, essa prática empresarial pode ser considerada reativa e não proativa, uma vez que na avaliação da satisfação do cliente o que se mede é a percepção do grau de aceitação do produto já desenvolvido pela empresa e adquirido pelos clientes.
Uma empresa com estratégia competitiva focada na satisfação dos clientes e com postura e práticas proativas deveria considerar a participação e a integração de clientes desde as etapas iniciais de seu processo de desenvolvimento de novos produtos.

A relevância da gestão da integração do cliente (IC) no processo de desenvolvimento de produto (PDP) está no fato de que, de maneira geral, a empresa tem capacidade para desenvolver soluções para as necessidades dos clientes e os clientes possuem informações sobre seus problemas e necessidades, para os quais buscam respostas, e que podem auxiliar no desenvolvimento mais adequado das soluções.

Normalmente, as reais necessidades dos clientes se encontram implícitas e são difíceis de serem expressas 
por eles, por exemplo, pelos métodos tradicionais de pesquisa de mercado (GRIFFIN; PRICE; VOJAK, 2009). No desenvolvimento de novos produtos, von Hippel (2001) propõe a interação entre a empresa fabricante e seus clientes em um mesmo espaço físico, ou geográfico, para alcançar uma boa solução e que satisfaça as necessidades implícitas e explícitas dos clientes. Gassmann, Sandmeier e Wecht (2006) consideram que o envolvimento do cliente deve ser visto como meio de aprendizagem organizacional e de melhoria da competitividade da empresa.

O tema integração do cliente no PDP e outros semelhantes, como inovação pelo usuário, codesenvolvimento (ou codesign), inovação participativa e aberta, têm merecido maior destaque no meio acadêmico na última década. Entretanto, Lettl (2007) observa que falta estruturação dos conteúdos estudados sobre o tema, assim como na definição de seus construtos e variáveis.

Para estruturar um tema de pesquisa, seu conteúdo e suas variáveis, é necessária uma ampla revisão bibliográfica sobre o mesmo. 0 método de revisão bibliográfica sistemática é um meio de gerar resultados generalizáveis e que podem ser usados para predições razoáveis de eventos futuros em relação ao tema (WEBSTER; WATSON, 2002). Para os autores, a pesquisa bibliográfica sistemática deve garantir que uma quantidade significativa das publicações pertinentes foi levantada. A revisão bibliográfica poderá ser concluída quando o pesquisador não encontrar mais novos conceitos e conhecimentos sobre o tema na sua pesquisa.

Neste artigo o objetivo não é criar um novo conhecimento teórico, mas fornecer um mapeamento, de amplo escopo, sobre o que tem sido pesquisado e publicado sobre o tema integração do cliente no PDP.

Os principais objetivos do artigo são: 1) identificar e classificar as publicações sobre o tema por ano, área de conhecimento, tipo de abordagem e de técnica de pesquisa; 2) analisar os principais tópicos e conteúdos tratados nas publicações sobre a integração do cliente no PDP; e 3) apresentar e organizar uma estrutura lógica de relacionamentos dos tópicos de pesquisa sobre a integração do cliente no PDP. A partir desses resultados, propõe-se temas para futuras pesquisas.

0 artigo está organizado em cinco partes. A parte dois explica o método de pesquisa utilizado. A três apresenta a síntese do levantamento bibliográfico e a classificação dos artigos. Na quatro são apresentados os resultados da análise bibliográfica e a classificação dos tópicos de pesquisa sobre integração do cliente no PDP. Em seguida, apresentam-se as considerações finais bem como recomendações para trabalhos de pesquisa sobre o tema.

\section{Método da pesquisa}

0 artigo pode ser considerado do tipo teóricoconceitual por ter como foco a realização de uma pesquisa de revisão bibliográfica sistemática, seguida de análise estruturada dos conteúdos publicados sobre o tema. A pesquisa pode ser classificada como exploratória e com abordagem quantitativa, uma vez que os dados e informações bibliográficas levantadas são classificados e analisados recorrendo-se a métodos quantitativos.

Adota-se um método de análise indutivo porque, a partir das informações levantadas nas publicações, sua análise e classificação, a estrutura lógica sobre os tópicos que envolvem o tema apresentada segue um critério de inferência. Quanto ao método de levantamento bibliográfico, pode ser definido como de revisão sistemática.

A revisão bibliográfica sistemática, para Pai et al. (2004), é uma abordagem de pesquisa confiável pela sua abrangência e apresentação explícita dos meios e resultados obtidos. Cook, Mulrow e Haynes (1997) e Pai et al. (2004) consideram que a revisão bibliográfica sistemática, em comparação com a revisão tradicional, inclui uma declaração clara do propósito da revisão, uma busca minuciosa de publicações, a avaliação crítica das principais publicações e a possibilidade de replicação do método de pesquisa.

Para Webster e Watson (2002), a revisão sistemática tem como objetivo gerar conhecimentos estruturados sobre um tema de pesquisa e pode ser usada para fazer predições razoáveis sobre o tema pesquisado.

A pesquisa foi conduzida em cinco etapas. $\mathrm{Na}$ primeira foram identificadas as palavras-chave nos trabalhos de tese de Kujala (2002), Janhager (2005) e Sandén (2007) sobre o tema de pesquisa, sendo elas: codevelopment, codesign, consumer focus, consumer involvement, consumer integration, consumer collaboration, consumer role, customer focus, customer involvement, customer integration, customer collaboration, user role, user focus, user involvement, user integration, user collaboration, junto às seguintes palavras-chave da área de desenvolvimento de produtos: product innovation e new product development.

$\mathrm{Na}$ segunda etapa, essas palavras-chave foram utilizadas para identificar e acessar as publicações nas bases de dados dos periódicos da CAPES (Coordenação de Aperfeiçoamento de Pessoal de Nível Superior): ACM, ACS, AIP, Blackwell, Cambridge University Press, Emerald, Gale, HighWire Press, IEEE, Nature, OECD, OVID, Oxford University Press, ProQuest, Sage, SciELO, Science Direct Online e Wilson. Essas bases de dados foram utilizadas devido a sua abrangência e ao acesso a artigos em texto completo. Nesta etapa, foram levantados 64 artigos sobre o tema, no período de 1980 a 2008. 
$\mathrm{Na}$ terceira etapa foi realizada a organização dos artigos por data de publicação. Após a leitura dos artigos (resumo, introdução e conclusões), foram observadas a existência de outras palavras-chave utilizadas nas publicações, tais como user innovation, customer competence e customer input. A partir da consideração dessas novas palavras-chave foram encontrados mais oito artigos relacionados ao tema, alcançando-se assim um total de 72 artigos. Esse tipo de abordagem de levantamento bibliográfico também é conhecido como levantamento bola de neve (snowball), conforme Yin (2002).

A quarta etapa consistiu na elaboração de fichamento de cada um dos artigos. Na quinta etapa, após a análise conjunta dos fichamentos, as informações relativas aos artigos foram organizadas em tabelas de síntese do tipo diagrama de afinidades, tendo como critério de classificação os tópicos mais citados nos artigos. Em seguida, os dados levantados foram analisados e foram propostos tópicos de pesquisa para evolução do conhecimento sobre o tema.

\section{Classificação dos artigos analisados}

Foram encontrados 72 artigos sobre integração do cliente no PDP, publicados em 39 periódicos no período de 1980 a 2008. Observou-se que 24 periódicos, dentre os 39 nos quais os artigos foram identificados, apresentavam apenas um artigo sobre o tema. Os periódicos que publicaram maior número de artigos sobre o tema foram: International Journal of Innovation Management, que publicou oito artigos (11,1\% do total de 72$)$ e The Journal of Product Innovation Management, que publicou 11 artigos (15,3\%).

A Tabela 1 apresenta os periódicos que mais publicaram artigos sobre integração do cliente no PDP no período analisado. Esses periódicos, juntos, acumulam 44 dos 72 artigos encontrados.

0 interesse sobre a integração do cliente no PDP, no meio acadêmico, aumentou significativamente nos últimos anos (LETTL, 2007). Essa afirmação pode ser justificada pelo aumento observado no número de artigos publicados nos últimos seis anos, conforme consta na Figura 1. Em 2002 houve aumento significativo em relação ao ano anterior, e a partir deste ano o aumento se manteve, com o ano de 2008 concentrando o maior número de publicações.

Uma segunda evidência do interesse pelo tema é a sua inclusão em livros sobre desenvolvimento de produtos. 0 livro da Product Development \& Management Association, Handbook of New Product Development, organizado por Kahn (2005), na sua segunda edição, ainda em 2005, incluiu capítulo denominado Interagindo com clientes no processo de
Tabela 1. Periódicos que mais publicaram artigos sobre IC no PDP.

\begin{tabular}{|l|c|}
\hline \multicolumn{1}{|c|}{ Periódico } & $\begin{array}{c}\text { Número de } \\
\text { publicações }\end{array}$ \\
\hline Journal of Product Innovation Management & 11 \\
\hline International Journal of Innovation Management & 8 \\
\hline International Journal of Technology Management & 4 \\
\hline European Journal of Innovation Management & 4 \\
\hline Management Science & 4 \\
\hline Harvard Business Review & 3 \\
\hline European Management Journal & 2 \\
\hline Industrial Marketing Management & 2 \\
\hline Journal of Engineering and Technology Management & 2 \\
\hline Journal of Service Marketing & 2 \\
\hline Technovation & 2 \\
\hline Total & 44 \\
\hline
\end{tabular}

OBS.: Em 28 outros periódicos foram encontrados um artigo por periódico. Período de 1980 a 2008.

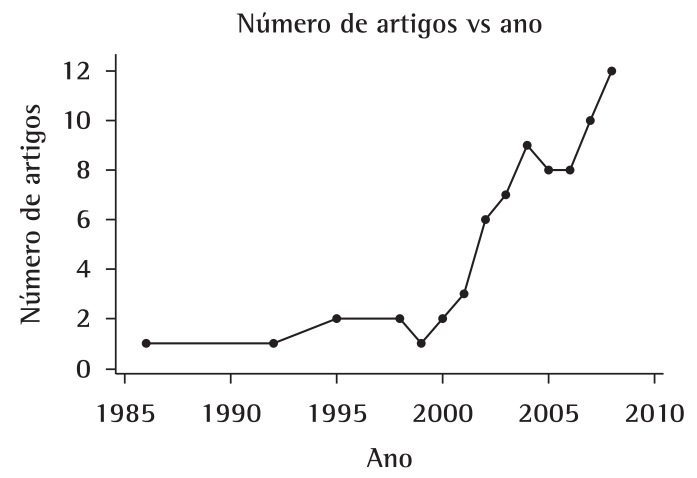

Figura 1. Número de artigos sobre IC no PDP por ano de publicação.

desenvolvimento de novos produtos. 0 mesmo aconteceu com o livro Handbook of New Product Development de Loch e Cavadias (2008), publicado pela Elseyver, no qual, dos 19 capítulos, três abordam a IC no PDP.

Para a classificação dos artigos, pelo tipo de pesquisa utilizada pelos autores, foi utilizada a tipologia de Voss et al. (2002) e de Creswell (2003). Os artigos identificados foram classificados em: estudo de caso; experimental; pesquisa-ação; survey; e teóricoconceitual. A Tabela 2 apresenta o resultado desta classificação. A pesquisa do tipo estudo de caso foi a mais utilizada pelos autores no período, representando $41 \%$ dos artigos, seguido por pesquisas do tipo survey (25\%) e por pesquisas teórico-conceituais (23\%).

Levando em consideração os critérios de classificação da abordagem de pesquisa apresentado por Creswell (2003), a Tabela 3 apresenta a classificação dos artigos em relação à sua abordagem: quantitativa descritiva, quantitativa preditiva, qualitativa descritiva, qualitativa preditiva e qualitativa-quantitativa. As abordagens de pesquisa qualitativa preditiva e quantitativa preditiva prevalecem nas publicações sobre o tema. 
Outro fator relevante na classificação dos artigos se refere à área de conhecimento dos principais autores. A integração do cliente no PDP é estudada por pesquisadores das áreas de conhecimento tais como: marketing, inovação, estratégia competitiva, qualidade, gestão do conhecimento e gestão da cadeia de suprimentos. A Tabela 4 mostra a classificação dos artigos por áreas de conhecimento dos autores. A área de conhecimento gestão da inovação tecnológica prevalece, seguida por marketing, gestão do conhecimento e estratégia competitiva.

Embora prevaleçam artigos de autores da área de gestão da inovação tecnológica, a integração do cliente no PDP pode ser considerada um tema multidisciplinar (KAUL1O, 1998; MATTHING; SANDÉN; EDVARDSSON, 2004). Vários artigos apresentam um enfoque multidisciplinar, como, por exemplo, o artigo Creating breakthroughs at 3M, de von Hippel, Thomke e Sonnack (1999). Os autores deste artigo são de diferentes áreas do conhecimento (gestão da tecnologia, marketing e psicologia). Essa multidisciplinaridade observa-se no conteúdo do artigo, que aborda bases teóricas provenientes da gestão da inovação tecnológica (tipos de inovação e processo de inovação); da área de marketing (alternativas de produtos e classificação de clientes) e da área de psicologia (observação do comportamento de clientes líderes).

Tabela 2. Classificação dos artigos por tipo de pesquisa realizado.

\begin{tabular}{cc}
\hline Tipo de pesquisa realizado & $\%$ \\
\hline Estudo de caso & 41 \\
Survey & 25 \\
Teórico-conceitual & 23 \\
Pesquisa-ação & 7 \\
Experimental & 4 \\
\hline
\end{tabular}

Tabela 3. Abordagens de pesquisa utilizadas nos artigos.

\begin{tabular}{cc}
\hline Abordagens & Número de artigos \\
\hline Qualitativa preditiva & 30 \\
Quantitativa preditiva & 24 \\
Qualitativa descritiva & 13 \\
Quantitativa descritiva & 3 \\
Qualitativa-quantitativa & 2 \\
Total & 72 \\
\hline
\end{tabular}

Tabela 4. Classificação dos artigos por área de conhecimento.

\begin{tabular}{cc}
\hline Área de conhecimento & $\#$ \\
\hline Gestão de inovação tecnológica & 42 \\
Marketing & 9 \\
Estratégia competitiva & 8 \\
Gestão de conhecimento & 8 \\
Gestão da cadeia de suprimentos & 3 \\
Gestão da qualidade & 2 \\
\hline
\end{tabular}

\section{Análise e classificação dos temas de pesquisa sobre integração do cliente no PDP}

Diversas definições são apresentadas para a integração do cliente no PDP, conforme citadas no Quadro 1. Levando-se em consideração a finalidade do estudo sobre o tema e os meios utilizados para atingir esse fim, as definições podem ser classificadas em dois grupos: como processo e como finalidade (criação de valor).

Na perspectiva de processo, diversas denominações são encontradas para essa integração: inovação participativa (BUUR; MATTHEUS, 2008), codesenvolvimento (NEALE; CORKINDALE, 1998) e interação com o cliente (KAUL10, 1998; GASSMANN, SANDMEIER; WECHT, 2006). Dessa forma, a integração do cliente pode ser vista como um processo no qual a empresa interage com seus clientes nas fases pré-desenvolvimento, desenvolvimento e pós-desenvolvimento.

Na perspectiva de criação de valor, no processo de desenvolvimento de produto, a integração do cliente representa uma abordagem diferente (von HIPPEL, 2005; PALS et al., 2008). Em um projeto de desenvolvimento em que os clientes são envolvidos em alto grau, o valor é criado interativamente para ambas as partes. Isso implica numa definição especial para o papel do cliente, uma perspectiva de relacionamento mais longo e duradouro entre as partes e uma oportunidade de gerar e adquirir novos conhecimentos (EDVARDSSON et al., 2006).

As teses de Kujala (2002), Janhager (2005) e Sandén (2007), os capítulos de livros revisados em Kahn (2005) e Loch e Kavadias (2008) e os 72 artigos levantados nesta pesquisa possibilitaram informações para obter os temas de pesquisa sobre a integração do cliente no PDP.

0 procedimento de análise foi verificar se o autor relaciona os temas de $\mathrm{IC}$ no objetivo do trabalho e/ ou nos resultados apresentados no trabalho. Por exemplo, o artigo de Lettl (2007) objetivou "estudar o papel do cliente e sua integração no desenvolvimento de projetos radicais e os benefícios para a empresa fabricante" 0 autor apresenta como resultado de sua pesquisa que os clientes possuem competências de mercado e tecnológicas e motivações para participar de desenvolvimentos de projetos radicais, além disso, mostra um processo para interação do cliente no PDP. Dessa forma, os temas da IC subtraídos do artigo foram: competência do cliente relacionada ao seu conhecimento das necessidades do mercado e seu conhecimento técnico do produto e atividades de interação, na qual o cliente faz parte da equipe do projeto. 
Quadro 1. Definições sobre integração do cliente no PDP.

\begin{tabular}{|c|l|}
\hline Autor & \multicolumn{1}{|c|}{ Definição } \\
\hline Kaulio (1998) & $\begin{array}{l}\text { A integração do cliente consiste nas atividades de interação entre os clientes e a equipe de desenvolvimento } \\
\text { de novos produtos. }\end{array}$ \\
\hline Neale e Corkindale (1998) & $\begin{array}{l}\text { Codesenvolvimento é o processo pelo qual as empresas que desenvolvem o novo produto e o cliente } \\
\text { tornam-se intimamente envolvidos no desenvolvimento, contribuindo com seus conhecimentos e } \\
\text { experiências. De maneira geral, os clientes fornecem conhecimentos para aplicação do produto e o } \\
\text { fabricante, o conhecimento sobre a tecnologia do produto e processo. }\end{array}$ \\
\hline Ritter e Walter (2003) & $\begin{array}{l}\text { Refere-se à abrangência ou amplitude de participação de clientes no processo de desenvolvimento de } \\
\text { produto, desde a geração da ideia até o teste do protótipo. }\end{array}$ \\
\hline Kujala (2003) & $\begin{array}{l}\text { A 1C pode ser entendida como um termo que descreve o contato direto entre os clientes e a empresa } \\
\text { fabricante durante o desenvolvimento do novo produto. }\end{array}$ \\
\hline Dahlsten (2004) & $\begin{array}{l}\text { A 1C no PDP pode ser definida como um processo de socialização no qual o conhecimento tácito do } \\
\text { cliente é obtido pela empresa. }\end{array}$ \\
\hline Kristensson, Gustafsson e \\
Archer (2004) & $\begin{array}{l}\text { A 1C no PDP oferece uma nova abordagem para aumentar a satisfação dos clientes pelo atendimento de } \\
\text { suas necessidades explícitas e implícitas. }\end{array}$ \\
\hline $\begin{array}{c}\text { Enkel, Kausch e Gassmann (2005) } \\
\text { e Gassmann e Wecht (2005) }\end{array}$ & $\begin{array}{l}\text { A 1C no PDP é um método, cada vez mais aplicado, que objetiva reduzir o risco de falha do produto no } \\
\text { seu lançamento no mercado. }\end{array}$ \\
\hline $\begin{array}{c}\text { Gassmann, Sandmeier e } \\
\text { Wecht (2006) }\end{array}$ & $\begin{array}{l}\text { A 1C é definida como a integração de clientes, clientes dos clientes ou intermediários de clientes nas } \\
\text { fases iniciais do processo de desenvolvimento de novos produtos, caracterizada pela participação ativa } \\
\text { dos clientes. }\end{array}$ \\
\hline Pals et al. (2008) & $\begin{array}{l}\text { A IC não significa pesquisar as necessidades dos clientes e criar um produto para eles, mas, sim, a } \\
\text { aprendizagem mútua entre a empresa fabricante e os clientes no PDP. }\end{array}$ \\
\hline
\end{tabular}

Outro exemplo é o trabalho de Enkel, Kausch e Gassmann (2005); Gassman e Wecht (2005), cujo objetivo de estudo foi "analisar o risco de integrar clientes em projetos de desenvolvimento de novos produtos". Os autores constataram que os benefícios de integrar clientes no PDP são maiores do que os riscos associados com sua integração, e que esses riscos podem diminuir se houver um bom critério de escolha dos clientes, definidos para cada tipo de projeto, escolha da técnica certa para conduzir a interação, escolha da infraestrutura necessária para a interação, escolha do número de clientes, papel do cliente e outras. Dessa maneira, os temas da IC subtraídos do artigo foram: critério de escolha do cliente, especificamente o Lead-Users; atividades de interação, que considera o cliente parte da equipe de projeto; e benefícios da IC no PDP, especificamente por reduzir as incertezas e riscos dos novos produtos e apresentar ideias para projetos radicais.

$\mathrm{Na}$ análise de cada fonte bibliográfica foi criado um grupo de temas referentes à IC no PDP. Depois de concluída a análise de todas as fontes, um diagrama de afinidade foi utilizado para agrupar os temas obtidos por semelhança. Para Mizuno (1993), o diagrama de afinidade tem como objetivo reunir fatos e ideias acerca de áreas desconhecidas e inexploradas que estão em estado de desorganização.

Foi utilizado um cartão para cada tema de $\mathrm{IC}$. Os cartões foram agrupados e em seguida rotulados. Esse procedimento foi repetido três vezes com intervalo de uma semana. Como resultado foi possível agrupá-los nas seguintes dimensões: fundamentos para a integração do cliente no PDP; critérios para

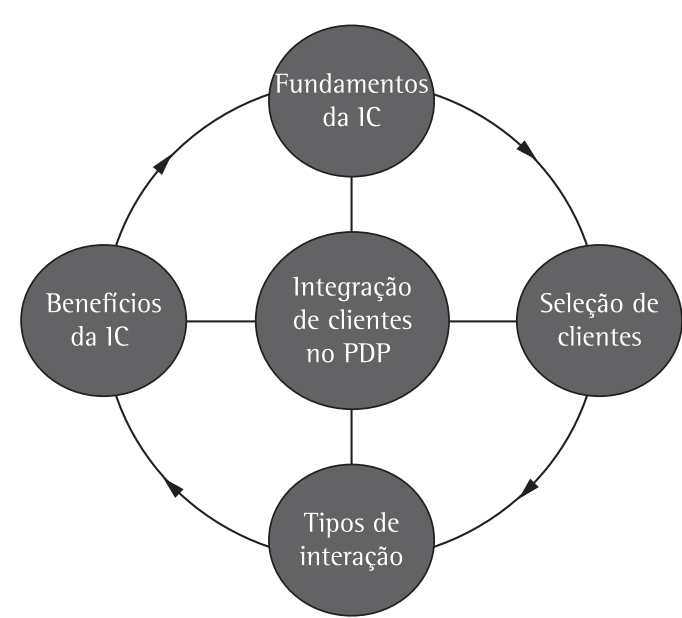

Figura 2. Dimensões de estudo sobre o tema da integração do cliente.

seleção dos clientes a serem envolvidos; tipos de interação entre os clientes e a empresa fabricante; e benefícios da integração do cliente. Os temas são considerados na descrição de cada uma das quatro dimensões definidas para representar a IC no PDP, conforme apresentado na Figura 2.

Essas dimensões representam conjuntos complementares de elementos necessários para gerenciar a IC no PDP e são relacionadas entre si. A primeira dimensão, fundamentos da $1 C$ no PDP, contempla os estudos que envolvem as razões pelas quais uma empresa deve envolver seus clientes no PDP. Uma vez que a empresa tiver clareza sobre 
esses fundamentos na sua estratégia, a dimensão seguinte contempla os estudos que citam os critérios para seleção de clientes. Depois do planejamento e selecionados os clientes para sua integração no PDP, há necessidade de uma terceira dimensão: tipo de interação entre a empresa e os clientes. A última dimensão considera os estudos que tratam dos benefícios obtidos pelas empresas com a $1 \mathrm{C}$ em um projeto de novo produto. Os benefícios obtidos se traduzem, posteriormente, em fundamentos ou na sua reformulação para a $\mathrm{IC}$, devido a que representam fatos reais que justificam o porque integrar os clientes nos projetos de novos produtos.

A seguir são apresentadas e analisadas cada uma dessas dimensões.

\subsection{Fundamentos para a integração do cliente no PDP}

0 sucesso comercial de um novo produto depende de muitos fatores, sendo um dos principais sua aceitação no mercado (PRAHALAD; RAMASWAMY, 2000; COOPER, 2005; GRIFFIN; PRICE; VOJAK, 2009). Aumentar a probabilidade de aceitação do novo produto exige a realização de atividades específicas durante o PDP, principalmente de pesquisas de mercado, com a finalidade de entender as reais necessidades do mercado. Na maioria das vezes, as necessidades dos clientes estão implícitas, o que torna complexo identificar claramente essas necessidades. Dessa forma, algumas empresas utilizam práticas como observação do comportamento do cliente no seu ambiente de trabalho e realização de entrevistas aprofundadas com a finalidade de entender essas necessidades. Outras empresas investem na integração de clientes nas fases iniciais do PDP, para melhor captarem as necessidades do mercado. Como consequência, um dos principais fundamentos para a IC no PDP é o conhecimento dos clientes sobre as necessidades do mercado, sejam elas implícitas ou explicitas.

Outro fundamento é o conhecimento tecnológico que determinados clientes possuem, principalmente em contexto de negócios B2B - Business to Business (GRUNER; HOMBURG, 2000; von HIPPEL, 2005). Em determinados setores industriais, como no caso dos fabricantes de chips, de equipamentos médicos e de instrumentos de laboratório, os clientes, além de possuírem necessidades de novos equipamentos ou de melhorias nos equipamentos já existentes, possuem conhecimentos técnicos e podem contribuir no projeto do novo produto. Para o desenvolvimento de equipamentos médicos, que envolvem conhecimento tecnológico e conhecimento específico de profissionais da área de saúde, são os médicos, como clientes, que contribuem no desenvolvimento dos novos produtos (LETTL, 2007; GONZALEZ et al., 2008).

Com o avanço das tecnologias da informação e do maior nível de profissionalismo dos clientes, relacionados à sua formação, cada vez mais os clientes detêm conhecimentos tecnológicos. Gassmann e Wetch (2005), Shah e Robinson (2006) e Raasch, Herstatt e Lock (2008) consideram que a participação dos clientes tem influência positiva no desempenho de projetos de novos produtos, não somente pelo conhecimento sobre o mercado mas, também, com o aporte de conhecimento técnico.

Do ponto de vista do marketing, outro fundamento se refere ao relacionamento que a empresa estabelece e realiza com seus clientes ao envolvê-los em projetos de novos produtos. Segundo Gruner e Homburg (2000), o relacionamento com os clientes por meio de canais de ouvidoria para sugestões e reclamações, entrevistas e consideração de suas ideias contribui para consolidar nos clientes uma posição (status) de importância que se traduz em satisfação e lealdade desses clientes para com a empresa. 0 CRM - Customer Relationship Management parte do pressuposto de que a empresa fabricante deve se relacionar e conhecer bem seus clientes e que esse relacionamento deve ser aprofundado e duradouro.

Sandén (2007) identificou um grupo de justificativas nas quais as empresas se baseiam para integrar seus clientes no PDP: os clientes são inovadores; a integração do cliente melhora o desempenho técnico do produto; a integração do cliente reduz o tempo total de desenvolvimento; a integração do cliente contribui para uma boa estratégia de marketing; a integração do cliente cria produtos e serviços de fácil uso. Esses resultados podem ser agrupados em três fundamentos para a IC no PDP: conhecimento das necessidades do mercado, conhecimento técnico do produto e manutenção de um bom relacionamento com os clientes.

A Tabela 5 apresenta os trabalhos publicados classificando-os por fundamentos para a IC no PDP.

Embora todos os artigos analisados considerem que os clientes apresentam conhecimento sobre 0 mercado, esse conhecimento, na maioria das vezes, se encontra implícito. Para explicitar esse conhecimento são necessárias técnicas de observação do cliente no seu ambiente real de trabalho (LEONARD-BARTON, 1998) e ou técnicas apropriadas de entrevista (GRIFFIN, PRICE; VOJAK, 2009) e/ou reuniões de trabalho em conjunto (von HIPPEL; KATZ, 2002).

O significativo número de artigos (42) que citam que os clientes possuem conhecimento técnico sobre o produto é explicado pela maior disponibilidade de 
Tabela 5. Fundamentos para a integração do cliente.

\begin{tabular}{|c|c|l|}
\hline Fundamento & $\begin{array}{c}\text { Número de } \\
\text { artigos }\end{array}$ & \multicolumn{1}{c|}{ Principais artigos } \\
\hline $\begin{array}{c}\text { Conhecimento das } \\
\text { necessidades do mercado }\end{array}$ & 72 & $\begin{array}{l}\text { Prahalad e Ramaswamy (2000); Ulwick (2002); Kujala (2003); Callahan e Lasry (2004); Dahlsten } \\
\text { (2004); Jepessen (2005); Lagrosen (2005); Su, Chen e Sha (2006); Franke, von Hippel e Schireier } \\
\text { (2006); Matthing, Kristensson e Gustafsson (2006); Shah e Robinson (2007); Lettl (2007); } \\
\text { Heiskanen et al. (2007); Kim e Bae (2008); Nambisan e Nambisan (2008); Warnke, Weber e } \\
\text { Leitner (2008); Braun e Herstatt (2008) }\end{array}$ \\
\hline $\begin{array}{c}\text { Conhecimento técnico do } \\
\text { produto pelos clientes }\end{array}$ & 42 & $\begin{array}{l}\text { Gruner e Homburg (2000); Luthje (2004); Lagrosen (2005); Luthje, Herstatt e von Hippel (2005); } \\
\text { Gassmann, Sandmeier e Wecht (2006); Su, Chen e Sha (2006); Shah e Robinson (2006); Lettl } \\
\text { (2007); Raasch, Herstatt e Lock (2008); Janssen e Dankbaar (2008); Buur e Matthews (2008); } \\
\text { Franke, Keinz e Schreier (2008) }\end{array}$ \\
\hline $\begin{array}{c}\text { Manutenção de um bom } \\
\text { relacionamento entre a } \\
\text { empresa e seus clientes }\end{array}$ & 16 & $\begin{array}{l}\text { Burton e Patterson (1999); Gruner e Homburg (2000); Goffin e New (2001); Bonner e Walker Junior } \\
\text { (2004); Lundkvist e Yaklef (2004); Berger et al. (2005); Lau, Yam e Tan (2007); Lam, Chin e Pun } \\
\text { (2007); Rowley, Kupiec-Teahan e Leeming (2007); Schreier e Prugl (2008); Kim e Bae (2008) }\end{array}$ \\
\hline
\end{tabular}

informação na sociedade, nos últimos anos, sobre produtos e o aumento do profissionalismo dos clientes em todos os setores econômicos.

Um terceiro fundamento, embora com significância média nas citações, se refere à busca da lealdade dos clientes por meio de programas de relacionamento. Pesquisas de Reichheld, Markey e Opton (2000) fundamentam a relevância da busca de lealdade dos clientes e seu impacto no resultado financeiro da empresa como um indicador de sustentabilidade financeira.

\subsection{Critérios para seleção de clientes}

Algumas publicações analisam os critérios utilizados na seleção de clientes que participam em projetos de novos produtos. Alam (2005) apresenta três critérios que deveriam ser considerados para a seleção de clientes.

Primeiro, uma empresa pode realizar interação com clientes com os quais já mantenha uma relação histórica de parceria, devido à confidencialidade das informações que envolvem o desenvolvimento de um novo produto. Um gerente de produto necessita confiar que os clientes parceiros manterão sigilo sobre as informações consideradas críticas e confidenciais. Um cliente parceiro também necessita mostrar seu compromisso de realizar com eficiência as atividades sob sua responsabilidade no PDP. Betts (1994 apud ALAM, 2005) cita o caso da Boeing no desenvolvimento do seu modelo de aeronave 777 , no qual, no início do desenvolvimento, houve a participação de oito diferentes empresas clientes (companhias aéreas) e como, mais tarde, interagiu mais ativamente com três de seus clientes parceiros: United Airlines, British Airways e All Nippon Airlines.

Segundo, interagir com clientes com capacidade inovadora. A característica inovadora do cliente pode ser captada pela empresa de diferentes formas: discussão de maneira informal de necessidades de mercado entre clientes e a equipe de projeto; análise das reclamações dos clientes sobre produtos existentes; discussão de ideias de novos produtos com o pessoal de vendas. Alam (2005) cita uma empresa que introduziu no mercado um cortador de grama que dispensa o uso das mãos, funciona à bateria e não precisa de pessoa para o manipular. A empresa teve a ideia quando um cliente da terceira idade reclamou sobre quão extenuante era cortar a grama com uma máquina manual e revelou que desejava uma máquina que fizesse o trabalho automaticamente, porém que esta deveria apresentar custo menor do que um cortador de grama tradicional. Em geral, os clientes que geram ideias de inovações tendem a conhecer o que propõem e podem articular de forma clara suas necessidades e ideias.

Terceiro, os clientes Lead-Users são a principal fonte de oportunidades para o desenvolvimento de produtos inovadores e lucrativos. 0 estudo de von Hippel (1986) tornou-se referência para o uso de critério de seleção de clientes participantes. 0 autor distingue entre clientes comuns e clientes Lead-Users. Os clientes Lead-Users têm duas características: 1) apresentam necessidades reais que se tornarão, no futuro, gerais no mercado e 2) esperam se beneficiar substancialmente com a obtenção de uma solução para suas necessidades.

Outros critérios para escolha dos clientes são apresentados por Bonner e Walker Junior (2004) e por Kristensson, Gustafsson e Archer (2004). Esses autores propõem que para otimizar a integração do cliente nas atividades do PDP, os fabricantes conheçam a representatividade do cliente no mercado, o esforço inovador do cliente, a disponibilidade do cliente para cooperar e a capacidade do cliente de manter sigilo das informações.

Outros critérios encontrados na bibliografia são: tamanho da demanda do cliente, atratividade financeira do cliente, ser cliente formador de opinião no segmento de mercado, ter reputação, conhecimento tecnológico, conhecimento e experiência passada em codesenvolvimento. 
Considerando os critérios de seleção de clientes citados, eles podem ser resumidos em: clientes com características Lead-Users; com alto poder aquisitivo; com competência técnica e inovadora; com disponibilidade para participar do projeto; com disponibilidade para divulgar o produto; com competência para testar o novo produto; e ser cliente formador de opinião.

Com base nesses critérios, os 72 artigos foram analisados e agrupados (Tabela 6).

Esses critérios de seleção de clientes podem ser considerados complementares. Por exemplo, quando um projeto representa uma mudança radical há necessidade de selecionar clientes Lead-Users para sua participação nas primeiras fases do desenvolvimento (critério citado em 32 artigos). Já para as últimas fases, como teste do protótipo, há necessidade de selecionar clientes com competência para testar o novo produto (citado em cinco artigos).

Também são destacados: disponibilidade do cliente para participar do projeto (29 artigos); potencial para divulgação do novo produto no mercado (11 artigos). Lettl (2007) observou esses critérios em empresas fabricantes de equipamentos médico-hopitalares.

0 critério poder aquisitivo do cliente, embora citado em sete artigos, não tem representatividade significativa em relação aos demais.

\subsection{Tipos de interação com os clientes}

Dependendo do tipo de projeto, do perfil do cliente e da fase do desenvolvimento do produto, a interação entre a empresa e seus clientes poderá ser de diferente tipo (EDVARDSSON et al., 2006). Para isso, a infraestrutura oferecida pelo fabricante para as atividades de participação do cliente torna-se fundamental, desde a disponibilidade de ambientes físicos até o uso de ambientes virtuais para a efetivação da interação.

Alam (2005) realizou um levantamento sobre as formas de interação entre uma empresa e seus clientes nas atividades consideradas importantes para o PDP. 0 autor identificou os seguintes tipos de interação: entrevistas em profundidade; brainstormings; integração do cliente na equipe de projeto; realização de painéis e participação dos clientes em grupos de discussão do produto; observação do comportamento do cliente no seu ambiente real de trabalho; reuniões informais em encontros não esperados; visitas a clientes; e interação por meio da internet.

Outros tipos de interação incluem: troca de e-mails (ROWLEY; KUPIEC-TEAHAN; LEEMING, 2007); entrevistas individuais (PALS et al., 2008); entrevistas em grupo (GRIFFIN, 1997); testes de protótipos em conjunto (SHAH; ROBINSON, 2006); reuniões virtuais (DAHAN; HAUSSER, 2002; KIM; BAE, 2008); e reuniões presenciais (HEISKANEN et al., 2007).

Os tipos de interação identificados nas publicações foram agrupados em: reuniões formais (entrevista individual, grupos focados, painéis de clientes, ensaios e testes em conjunto, participação em testes de protótipos); reuniões informais (por exemplo, encontros em almoços); interação on-line (reuniões virtuais); integração como membro da equipe de projeto (clientes que integram a equipe de projeto); observação do cliente (observação do comportamento do cliente no seu ambiente real de uso do produto); e por

Tabela 6. Critérios para seleção de clientes.

\begin{tabular}{|c|c|c|}
\hline Critério & $\begin{array}{l}\text { Número } \\
\text { de artigos }\end{array}$ & Principais fontes \\
\hline Cliente Lead-User & 32 & $\begin{array}{l}\text { von Hippel (1998); von Hippel, Thomke e Sonnack (1999); Olson e Bakke (2001); von Hippel } \\
\text { (2001); Lilien et al. (2002); Ulwick (2002); von Hippel e Katz (2002); Enkel, Kausch e Gassman } \\
\text { (2005); Gassmann e Wecht (2005); Gassmann, Sandmeier e Wecht (2006); Lettl (2007); Schreier e } \\
\text { Prugl (2008); Franke, Keinz e Schreier (2008); Bilgram, Brem e Voigt (2008) }\end{array}$ \\
\hline $\begin{array}{l}\text { Disponibilidade do cliente } \\
\text { para participar do projeto } \\
\text { do novo produto }\end{array}$ & 29 & $\begin{array}{l}\text { Dahan e Hauser (2002); Magnusson (2003); Luthje (2004); Franke e Piller (2004); Lundkvist e } \\
\text { Yaklef (2004); Matthing, Lettl (2007); Bilgram, Brem e Voigt (2008); Raasch, Herstatt e Lock } \\
\text { (2008); Janssen e Dankbaar (2008) }\end{array}$ \\
\hline $\begin{array}{l}\text { Cliente com competência } \\
\text { técnica e inovadora }\end{array}$ & 11 & $\begin{array}{l}\text { Olson e Bakke (2001); Brockhoff (2003); Bonner e Walker Junior (2004); Kristensson, Gustafsson } \\
\text { e Archer (2004); Luthje, Herstatt e von Hippel (2005); Jepessen (2005); Jacob (2006); Lettl (2007) }\end{array}$ \\
\hline $\begin{array}{l}\text { Cliente com disponibilidade } \\
\text { para divulgação do novo } \\
\text { produto }\end{array}$ & 11 & $\begin{array}{l}\text { Gruner e Homburg (2000); Bonner e Walker Junior (2004); May-Plumlee e little (2006); Rowley, } \\
\text { Kupiec-Teahan e Leeming (2007); Lettl (2007); Franke, Keinz e Schreier (2008); Braun e Herstatt (2008) }\end{array}$ \\
\hline $\begin{array}{l}\text { Cliente com alto poder } \\
\text { aquisitivo }\end{array}$ & 7 & $\begin{array}{l}\text { Gruner e Homburg (2000); Dahlsten (2004); Lundkvist e Yaklef (2004); Matthing, Kristensson e } \\
\text { Gustafsson (2006); Raasch, Herstatt e Lock (2008) }\end{array}$ \\
\hline $\begin{array}{l}\text { Cliente com competência } \\
\text { para testar o novo produto }\end{array}$ & 5 & Sioukas (1995); Gruner e Homburg (2000); Brockhoff (2003); Pals et al. (2008) \\
\hline $\begin{array}{l}\text { Cliente formador de } \\
\text { opinião no segmento de } \\
\text { mercado }\end{array}$ & 4 & Shah e Robinson (2006); Anderson, Rosenqvist e Ashrafi (2007) \\
\hline
\end{tabular}


correspondência (interação pelo telefone, por meio de correio e troca de e-mails).

0 agrupamento das publicações por tipos de interação está detalhado na Tabela 7.

A integração de clientes nas equipes de projeto (citada em 29 artigos) é a que predomina nos artigos. Essa integração dos clientes pode ser especifica para uma determinada fase do projeto, ocorrer em várias fases ou durante todo o processo de desenvolvimento do projeto.

A segunda maior citação do tipo de interação, interação on-line, advém da evolução das tecnologias de informação e de comunicação. Esse tipo de interação é considerada de maior relevância, em termos de custos, quando a empresa tem como mercado-alvo clientes localizados em diferentes regiões geográficas (DAHAN; HAUSER, 2002). Por outro lado, requer da empresa disponibilidade de infraestrutura para essa finalidade e capacitação em tecnologias de informação pela equipe do projeto.
Os tipos de interação menos citados foram reuniões informais e observação do cliente no seu ambiente real de uso do produto. A observação de clientes em seu ambiente de trabalho, segundo Griffin, Price e Vojak (2009), é utilizada como complemento para levantar informações de problemas e/ou necessidades dos clientes.

\subsection{Benefícios da integração do cliente no PDP}

Diversos ganhos podem ser obtidos com a integração de clientes no PDP. A Tabela 8 apresenta os tipos de benefícios potenciais citados nos artigos que as empresas podem obter ao envolver os clientes em projetos de desenvolvimento de novos produtos.

Os diferentes benefícios que uma empresa pode obter ao envolver clientes no PDP podem ser agrupados em: maior eficácia do produto desenvolvido e maior eficiência no desenvolvimento do projeto. 0 primeiro grupo abrange a redução das incertezas no lançamento

Tabela 7. Tipos de interação nas atividades de IC no PDP.

\begin{tabular}{|c|c|c|}
\hline $\begin{array}{c}\text { Tipos de } \\
\text { interação }\end{array}$ & $\begin{array}{c}\text { Número de } \\
\text { artigos }\end{array}$ & Principais artigos \\
\hline $\begin{array}{c}\text { Como membro } \\
\text { da equipe de } \\
\text { projeto }\end{array}$ & 29 & $\begin{array}{l}\text { von Hippel, Thomke e Sonnack (1999); Olson e Bakke (2001); Ulwick (2002); Lilien et al. (2002); Magnusson } \\
\text { (2003); Enkel, Kausch e Gassmann (2005); Gassman e Wecht (2005); Gassmann, Sandmeier e Wecht (2006); } \\
\text { Franke, von Hippel e Schireier (2006); Lettl (2007); Schreier e Prugl (2008); Buur e Matthews (2008) }\end{array}$ \\
\hline $\begin{array}{c}\text { Interação } \\
\text { on-line }\end{array}$ & 26 & $\begin{array}{l}\text { von Hippel (2001); Dahan e Hauser (2002); von Hippel e Katz (2002); Tomke e von Hippel (2002); Franke e } \\
\text { Siller (2004); Jepessen (2005); Rowley, Kupiec-Teahan e Leeming (2007); Kim e Bae (2008); Franke, Keinz e }\end{array}$ \\
\hline $\begin{array}{c}\text { Reuniões } \\
\text { formais }\end{array}$ & 22 & $\begin{array}{l}\text { Sioukas (1995); von Hippel (1998); Lilien et al. (2002); Dahan e Hauser (2002); Franke e Piller (2004); } \\
\text { Mascarenhas, Kesavan e Bernacchi (2004); Pals et al. (2008); Buur e Matthews (2008) }\end{array}$ \\
\hline $\begin{array}{c}\text { Por } \\
\text { correspondência }\end{array}$ & 12 & $\begin{array}{l}\text { Magnusson (2003); Franke e Piller (2004); Rowley, Kupiec-Teahan e Leeming (2007); Kim e Bae (2008); } \\
\text { Bilgram, Brem e Voigt (2008); Raasch, Herstatt e Lock (2008) }\end{array}$ \\
\hline $\begin{array}{c}\text { Observação } \\
\text { do cliente }\end{array}$ & 3 & Deschamps e Nayak (1997); Alam e Perry (2002); Griffin, Price e Vojak (2009) \\
\hline $\begin{array}{c}\text { Reuniões } \\
\text { informais }\end{array}$ & 2 & Nambisan e Nambisan (2002); Alam e Perry (2002) \\
\hline
\end{tabular}

Tabela 8. Benefícios da integração de cliente.

\begin{tabular}{|c|c|c|}
\hline Benefícios & $\begin{array}{l}\text { Números } \\
\text { de artigos }\end{array}$ & Principais artigos \\
\hline ldeias para inovação radical & 32 & $\begin{array}{l}\text { von Hippel (1986); von Hippel (2001); Lilien et al. (2002); Magnusson (2003); } \\
\text { Kristensson, Gustafsson e Archer (2004); Matthing, Sandén, Edvardsson (2004); } \\
\text { Enkel, Kausch e Gassmann (2005); Gassmann e Wecht (2005); Heiskanen et al. } \\
\text { (2007); Lettl (2007); Shah e Robinson (2007); Schreier e Prugl (2008) }\end{array}$ \\
\hline $\begin{array}{l}\text { Redução de incertezas e riscos dos novos } \\
\text { produtos }\end{array}$ & 32 & $\begin{array}{l}\text { Gales e Mansour-Cole (1995); Dahlsten (2004); Kristensson, Gustafsson e Archer } \\
\text { (2004); Matthing, Sandén, Edvardsson (2004); Enkel, Kausch e Gasussman } \\
\text { (2005); Gassmann e Wecht (2005); Enkel, Perez-Freije e Gassmann (2005); } \\
\text { Gassman, Sandmeier e Wecht (2006); Heiskanen et al. (2007); Schreier e Prugl } \\
\text { (2008) }\end{array}$ \\
\hline 1deias para inovação incremental & 11 & von Hippel (2001); Kujala (2003); Brockhoff (2003); Shah e Robinson (2007) \\
\hline Redução de custo de desenvolvimento & 9 & $\begin{array}{l}\text { von Hippel (1998); von Hippel e Katz (2002); Brockhoff (2003); Franke e Piller } \\
\text { (2004); Jepessen (2005) }\end{array}$ \\
\hline Aumento do nível de parceria & 6 & Lau, Yam e Tam (2007); Shah e Robinson (2007); Schreier e Prugl (2008) \\
\hline Maior produtividade da equipe do projeto & 3 & Hersttat e von Hippel (1992); Chen e Yan (2007); Buur e Matthews (2008) \\
\hline Redução do número de mudanças no projeto & 3 & Hersttat e von Hippel (1992); Kujala (2003); Buur e Matthews (2008) \\
\hline
\end{tabular}


do novo produto, assim como a obtenção de ideias para projetos radicais e incrementais. 0 segundo grupo contempla a redução dos custos associados ao desenvolvimento do projeto, redução do número de mudanças no projeto e ganhos por maior produtividade da equipe do projeto.

Embora Becker e Peters (1998) considerem como desvantagem da IC o risco de gerar conflitos entre a empresa fabricante e seus clientes, por questões de propriedade intelectual, Enkel, Kausch e Gassman (2005); Gassmann e Wecht (2005) consideram que esse tipo de risco pode ser reduzido pelo gerenciamento dessa integração e concluem que o risco de integrar clientes é menor em comparação ao risco da sua não integração.

\section{Considerações finais}

A integração de clientes no PDP, frequentemente citada como relevante nas áreas de conhecimento e estratégia competitiva, inovação tecnológica, qualidade e marketing, até a década de 1990 despertou relativamente pouco interesse da comunidade acadêmica, tendo-se como referência o número de publicações de artigos e livros que abordam essa questão. Na primeira década do século XXI esse cenário de interesse científico mudou, com aumento significativo do número de artigos publicados e pela incorporação do tema em capítulos de livros sobre gestão do PDP.

Quanto à definição da integração de clientes no PDP, ainda não se tem uma conceituação consensual nas publicações e entre os autores que abordam o tema. Os autores que apresentam conceituações sobre o tema, pertencentes a diferentes áreas do conhecimento, apresentam definições que incluem: transferência de informação; interação entre a empresa e seus clientes em atividades do PDP; criação de valor; e processo de socialização do conhecimento no desenvolvimento de produto.

Neste artigo sugere-se a definição de IC no PDP como uma estratégia de desenvolvimento de produto de uma empresa cuja diretriz básica é a busca e solução de necessidades do mercado. A prática da IC nas empresas pode ser classificada por níveis de maturidade, desde um tipo de integração que considera o cliente emissor de opiniões (por meio de entrevistas), como fonte de observação do seu comportamento (observação no ambiente que será utilizado o produto), até como participante ativo na equipe de projeto.

Em todos os artigos analisados argumenta-se que a IC é uma abordagem que auxilia na melhor identificação das reais necessidades do mercado. Como citado por alguns autores (von Hippel, Thomke e Sonnack (1999); Prahalad e Ramaswamy (2000); Edvardsson et al. (2006)), as técnicas de pesquisa de mercado tradicionais, por meio das quais a área de marketing capta informações do mercado e as traduz e repassa para as equipes de projeto, são criticadas por dois motivos básicos. Primeiro, pelo fato de que na maioria das vezes as necessidades dos clientes se encontram implícitas e as técnicas tradicionais de pesquisa de mercado são deficientes para captar necessidades implícitas. Segundo, a existência de um intermediário entre a fonte de informação sobre as necessidades do mercado (clientes) e os usuários dessa informação (equipes de desenvolvimento do produto) aumenta a probabilidade de erro, pelas dificuldades na interpretação ou conversão das informações sobre as necessidades do mercado.

Em alguns artigos (OLSON; BAKKE, 2001; BROCKHOFF, 2003; BONNER; WALKER JUNIOR, 2004; KRISTENSSON; GUSTAFSSON; ARCHER, 2004; LUTHJE, HERSTATT; von HIPPEL, 2005; JEPESSEN, 2005; JACOB, 2006; LETTL, 2007), se argumenta que, além de criar um relacionamento de parceria com os clientes, a integração de clientes nos projetos de novos produtos proporciona à empresa fabricante a obtenção de conhecimento técnico sobre o produto. Esses benefícios são mais significativos em ambientes de negócio B2B.

$\mathrm{O}$ agrupamento dos temas de pesquisa em quatro dimensões ajuda a compreender o tema da IC no PDP de maneira especifica quando se objetiva gerenciar essa integração. Entretanto, é necessário que as características dessas dimensões e seus desdobramentos sejam aprofundados com pesquisas empíricas.

Como resultado desta pesquisa bibliográfica apresentam-se as seguintes proposições para pesquisa:

1) As atividades de IC no PDP permite às empresas conhecer melhor as necessidades do mercado, a aquisição de conhecimento técnico do produto na perspectiva dos clientes e a obtenção de um melhor relacionamento com seus clientes;

2) Os critérios da seleção de clientes para a integração no PDP dependem do segmento de mercado na qual a empresa atua;

3) 0 tipo de interação entre a empresa e seus clientes varia nas diferentes fases do PDP;

4) 0 grau de IC no PDP varia de acordo com o grau de novidade do novo produto.

Recomenda-se aos interessados no tema de IC no PDP pesquisar, por meio do estudo de casos e de pesquisas de levantamento cada uma das dimensões dessa integração. De maneira específica, temas para futuras pesquisas deveriam procurar responder perguntas como: 
Qual a relação entre o grau de incerteza do projeto de um novo produto e o nível de IC no projeto? Que incentivos têm os clientes para participar de projetos de novos produtos? Como deveriam ser planejadas as atividades de IC no PDP? Em que circunstâncias os clientes se sentem mais à vontade para compartilhar suas ideias e conhecimentos de novos produtos? Quais métodos são mais eficientes para identificar e compreender as necessidades latentes dos clientes?

A busca de resposta a essas questões permitirá gerar informações para estruturar um modelo de gestão da IC no PDP, necessário para obter eficácia e eficiência nessa integração e para os novos produtos desenvolvidos.

\section{Referências}

ALAM, 1. Interacting with customers in the new product development process. In: KAHN, K. B. The PMDA handbook of new product development. 2nd ed. New Jersey: John Wiley \& Sons, 2005. p. 249-262.

ALAM, 1.; PERRY, A. A customer-oriented new service development. Journal of Service Marketing, v. 16, n. 2, p. 515-534, 2002. http://dx.doi.org/10.1108/08876040210443391

ANDERSON, P.; ROSENQVIST, C.; ASHRAFl, 0. Mobile innovations in healthcare: customer involvement and the co-creation of value. International Journal of Mobile Communications, v. 5, n. 4, 2007. http://dx.doi.org/10.1504/1JMC.2007.012786

BECKER, W.; PETERS, J. R\&D-competition between vertical corporate networks: market structure and strategic R\&D-spillovers. Economics of Innovation \& New Technology, v. 6, n. 1, p. 51-71, 1998. http://dx.doi.org/10.1080/10438599800000013

BERGER, C. et al. Co-design modes of cooperation at the customer interface: learning from exploratory research. European Management Review, v. 2. p. 70-87, 2005. http://dx.doi. $\operatorname{org} / 10.1057 /$ palgrave.emr. 1500030

BILGRAM, V.; BREM, A.; VOIGT, K. User-centric innovations in new product development: systematic identifications of lead users harnessing interactive and collaborative onlinetools. International Journal of Innovation Management, v. 12, n. 3, p. 418-458, 2008. http://dx.doi.org/10.1142/ S1363919608002096

BONNER, J. M.; WALKER JUNIOR, O. C. Selecting influential Business-to-Business customers in new product development: relational embeddedness and knowledge heterogeneity considerations. The Journal of Product Innovation Management, v. 4, n. 21, p. 155-169, 2004. http://dx.doi.org/10.1111/j.07376782.2004.00067.x

BRAUN, B.; HERSTATT, C. The freedom-fighters: how incumbent corporations are attempting to control user innovation. International Journal of Innovation Management, v. 12, n. 3, p. 543-572, 2008. http://dx.doi.org/10.1142/ S1363919608002059

BROCKHOFF, K. Customers' perspectives of involvement in new product development. International Journal of Technology Management, v. 26, n. 5-6, p. 464-481, 2003. http://dx.doi. org/10.1504/1JTM.2003.003418

BURTON, A.; PATTERSON, S. Integration of consumer and management in NPD. Journal of the Market Research Society, v. 41 , n. 1 , p. $61-74,1999$

BUUR, J.; MATTHEUS, B. Participatory innovation. International Journal of Innovation Management, v. 12, n. 3, p. 255-273, 2008. http://dx.doi.org/10.1142/S1363919608001996
CALLAHAN, J.; LASRY, E. The importance of customer in the development of very new products. $R \& D$ Management, v. 34, n. 2, p. 107-120, 2004. http://dx.doi.org/10.1111/j.14679310.2004.00327.x

CHENG, CHUN-HSIEN; YAN, W. An in-process customer utility prediction system for product conceptualization. Expert System with Applications, v. 34, n. 4, p. 2555-2567, 2007. http://dx.doi. org/10.1016/j.eswa.2007.04.019

COOK, D.; MULROW, C.; HAYNES, R. Systematic reviews: synthesis of best evidence for clinical decisions. Annals of Internal Medicine, v. 126, p. 376-80, 1997.

COOPER, R. New products: what separates the winners from the losers and what drive success. In: KAHN, K. The PMDA handbook of new product development. 2nd ed. New Jersey: John Wiley \& Sons, 2005. p. 3-28.

CRESWELL, J. W. Research design: qualitative and quantitative approaches. London: Sage, 2003.

DAHAN, E.; HAUSER, J. R. The virtual customer. The Journal of Product Innovation Management, v. 19, p. 332-353, 2002. http://dx.doi.org/10.1016/S0737-6782(02)00151-0

DAHLSTEN, F. Hollywood wives revisited: a study of customer involvement in the XC90 project at Volvo Cars. European Journal of Innovation Management, v. 7, n. 2, p. 141-149, 2004. http:// dx.doi.org/10.1108/14601060410534384

DESCHAMPS, J. P.; NAYAK, P. R. Produtos irresistíveis. Rio de Janeiro: Makron Books, 1997.

EDVARDSSON, B. et al. Involving customer in new service development. Sweden: Imperial College Press, 2006. http:// dx.doi.org/10.1142/9781860948893

ENKEL, E.; KAUSCH, C.; GASSMANN, O. Managing the risk of customer integration. European Management Journal, v. 23, n. 2, p. 203-213, 2005. http://dx.doi.org/10.1016/j. emj.2005.02.005

ENKEL, E.; PEREZ-FREIJE, J.; GASSMANN, 0. Minimizing market risks through customer integration in new product development: Learning from bad practice. Journal Compilation, v.14, n. 4, 2005.

FRANKE, N.; PILLER, F. Value creating by toolkits for user innovation and design: the case of the watch market. The Journal of Product Innovation Management, v. 21, p. 401-415, 2004. http://dx.doi.org/10.1111/j.0737-6782.2004.00094.x

FRANKE, N.; KEINZ, P.; SCHREIER, M. Complementing mass customization toolkits with user communities: how peer input improves customer self-design. The Journal of Product Innovation Management, v. 25, p. 546-559, 2008. http://dx.doi. org/10.1111/j.1540-5885.2008.00321.x

FRANKE, N.; von HIPPEL, E.; SCHREIER, M. Finding commercially attractive user innovations: a test of Lead-User theory. The Journal of Product Innovation Management, v. 23, p. 301-315, 2006. http://dx.doi.org/10.1016/0923-4748(95)00005-7

GALES, L.; MANSOUR-COLE, D. User involvement in innovations projects: toward an information processing model. Journal of Engineering and Technology Management, v. 12, p. 77-109, 1995. http://dx.doi.org/10.1016/0923-4748(95)00005-7

GASSMANN, 0.; WECHT, C. A strategic view on early customer integration into the innovation process. STRATEGIC MANAGEMENT SOCIETY ANNUAL INT. CONF. (SMS), 25., 2005. Orlando. Proceedings... 2005.

GASSMANN, O.; SANDMEIER, P.; WECHT, C. H. Extreme customer innovation in the front-end: learning from a new software paradigm. International Journal of Technology Management, v. 33, n. 1, p. 46-66, 2006. http://dx.doi.org/10.1504/ 1JTM.2006.008191

GOFFIN, K.; NEW, C. Customer support and new product development: an exploratory study. In: International Journal of Operations \& Production Management, v. 21, n. 3, p. 275-301, 2001. http:// dx.doi.org/10.1108/01443570110364605 
GONZALEZ, M. O. et al. 0 envolvimento do cliente no processo de desenvolvimento de produtos: estudo de casos no setor de equipamentos médico-hospitalares. ln: SIMPEP, 15., Bauru, 2008. Anais... 2008.

GRIFFIN, A. PDMA Research on new product development practices: updating trends and benchmarking best practices, Journal of Product Innovation Management, v. 14, n. 6, p. 429-458, 1997.

GRIFFIN, A.; PRICE, R.; VOJAK, B. More than just customer needs: how serial innovators develop a deep understanding of business customer' problem. In: INTERNATIONAL PRODUCT DEVELOPMENT MANAGEMENT CONFERENCE, 16., Enschede, Netherlands, 2009. Proceedings...

GRUNER, K. E.; HOMBURG, C. Does customer interaction enhance new product success? Journal of Business Research, n. 49, p. 1-14, 2000.

HEISKANEN, E. et al. User involvement in radical innovation: are consumers conservative? European Journal of Innovation Management, v. 10, n. 4, p. 489-509, 2007. http://dx.doi. org/10.1108/14601060710828790

HERSTATT, C.; von HIPPEL, E. From experience: Developing new product concepts via the lead user method: A case study in a "low-tech" field. Journal of Product Innovation Management, v. 9, n. 3, p. 213-221, 1992.

JACOB, F. Preparing industrial suppliers for customer integration. Industrial Marketing Management, v. 35, p. 45-56, 2006.

JANHAGER, J. User consideration in early stage of product development: theories and methods. 2005. $112 \mathrm{f}$. Tese (Doctor of Engineering in Machine Design)-Department of Machine Design, Royal Institute of Technology, Stockholm, 2005.

JANSSEN, K. L.; DANKBAAR, B. Proactive involvement of consumers in innovation: selecting appropriate techniques. International Journal of Innovation Management, v. 12, n. 3, p. 511-541, 2008. http://dx.doi.org/10.1142/S1363919608002047

JEPPESEN, L. B. User toolkits for innovation: consumers support each other. The Journal of Product Innovation Management, v. 22, p. 347-362, 2005. http://dx.doi.org/10.1111/j.07376782.2005.00131.x

KAHN, K. The PMDA handbook of new product development. 2nd ed. New Jersey: John Wiley \& Sons, 2005.

KAUL10, M. A. Customer, consumer and user involvement in new product development: a framework and a review of selected methods. Total Quality Management, v. 9, n. 1, p. 141-149, 1998. http://dx.doi.org/10.1080/0954412989333

$\mathrm{KIM}$, J. H.; BAE, Z. The role of online brand community in new product development: case studies on digital product manufacturers in Korea. International Journal of Innovation Management, v. 12, n. 3, p. 357-376, 2008. http://dx.doi. org/10.1142/S1363919608002011

KRISTENSSON, P.; GUSTAFSSON, A.; ARCHER, T. Harnessing the creative potential among users. The Journal of Product Innovation Management, v. 21, p. 4-14, 2004. http://dx.doi. org/10.1111/j.0737-6782.2004.00050

KUJALA, S. User studies: a practical approach to user involvement for gathering user needs and requirements. 2002. $61 \mathrm{f}$. Tese (Doctor of Philosophy)-Helsinki University of Technology, Finland, 2002.

KUJALA, S. User involvement: a review of the benefits and challenges. Behaviour \& Information Technology, v. 22, n. 1, p. 1-16, 2003. http://dx.doi.org/10.1080/01449290301782

LAGROSEN, S. Customer involvement in new product development: a relationship marketing perspective. European Journal of Innovation Management, v. 8, n. 4, p. 424-436, 2005. http:// dx.doi.org/10.1108/14601060510627803

LAM, P. K.; CHIN, K. S.; PUN, K. F. Managing conflict in collaborative product development: a supplier development. International Journal of Quality \& Reliability Management, v. 24, n. 9, p. 891-907, 2007. http://dx.doi. org/10.1108/02656710710826171
LAU, A. K. W.; YAM, R. C. M.; TAN, E. P. Y. Supply chain product co-development, product modularity and product performance. Industrial Management \& Data System, v. 107, n. 7, p. 1036-1065, 2007. http://dx.doi. org/10.1108/02635570710816739

LEONARD-BARTON, D. Nascentes do saber. Rio de Janeiro: Editora Fundação Getulio Vargas, 1998.

LETTL, C. User involvement competence for radical innovation. Journal of Engineering and Technology Management, v. 24 , p. 53-75, 2007. http://dx.doi.org/10.1016/j. jengtecman.2007.01.004

LILIEN, G. L. et al. Performance assessment of the Lead User ideageneration process for new product development. Management Science, v. 48, n. 8 , p. $1042-1059$, 2002. http://dx.doi. org/10.1287/mnsc.48.8.1042.171

LOCH, C. H.; CAVADIAS, S. Handbook of new product development management. Oxford: Elsevier, 2008.

LUNDKVIST, A.; YAKHLEF, A. Customer involvement in new service development: a conversational approach. Managing Service Quality, v. 14, n. 2-3, p. 249-257, 2004. http://dx.doi. org/10.1108/09604520410528662

LUTHJE, C. Characteristics of innovation users in a consumer good fiel: an empirical study of sporte-related product consumer. Technovation, v. 24, p. 683-695, 2004. http://dx.doi. org/10.1016/S0166-4972(02)00150-5

LUTHJE, C.; HERSTATT, C.; von HIPPEL, E. User-innovators and "local" information: the case of mountain biking. Research Policy, v. 34, p. 951-965, 2005. http://dx.doi.org/10.1016/j. respol.2005.05.005

MAGNUSSON, P.R. Benefits of involving users in service innovation. European Journal of Innovation Management, v. 6, n. 4, p. 228-238, 2003. http://dx.doi. org/10.1177/1094670503257028

MASCARENHAS, 0.; KESAVAN, R.; BERNACCHI, M. Customer valuechain involvement for co-creating customer delight. Journal of Consumer Marketing, v. 21, n. 7, p. 486-496, 2004. http:// dx.doi.org/10.1108/07363760410568707

MATTHING,J.;SANDÉN, B.;EDVARDSSON, B. Newservicedevelopment: learning from and with customers. International Journal of Service Industry Management, v. 15, n. 5, p. 479-498, 2004. http://dx.doi.org/10.1108/09564230410564948

MATTHING, J.; KRISTENSSON, P.; GUSTAFSSON, A. Developing successful technology-based services: the issue of identifying and involving innovative users. Journal of Service Marketing, v. 20, n. 5, p. 288-297, 2006. http://dx.doi. org/10.1108/09564230410564948

MAY-PLUMLEE, T.; LITTLE, T. J. Proactive product development integrating consumer requirements. International Journal of Clothing Sicnce and Technology, v. 18, n. 1, p. 53-66, 2006. http://dx.doi.org/10.1108/09556220610637512

MIZUNO, S. Gerência para melhoria da qualidade: as sete novas ferramentas de controle da qualidade. Rio de Janeiro: LTC, 1993.

NAMBISAN, S.; NAMBISAN, P. How to profit from a better virtual customer environment. MIT Sloan Management Review, v. 49, n. 3, p. 53-61, 2008.

NEALE, M.; CORKINDALE, D. Co-developing products: involving customers earlier and more deeply. Long Range Planning, v. 31, n. 3, p. 418-425, 1998. http://dx.doi.org/10.1016/S00246301(98)80008-3

OLSON, E. L.; BAKKE, G. Implementing the lead user method in a high technology firm: a longitudinal study of intentions versus actions. The Journal of Product Innovation Management, v. 18, p. 388-395, 2001. http://dx.doi.org/10.1016/S07376782(01)00111-4

$\mathrm{PAl}, \mathrm{M}$. et al. Systematic reviews and meta-analyses: an illustrated step-by-step guide. The National Medical Journal of India, v. 17 , n. 2 , p. $86-95,2004$. 
PALS, N. et al. Three approaches to take the user perspective into account during new product design. International Journal of Innovation Management, v. 12, n. 3, p. 275-294, 2008. http:// dx.doi.org/10.1142/S1363919608002023

PRAHALAD, C. K.; RAMASWAMY, V. Co-opting customer competence. Harvard Business Review, p. 79-87, 2000.

RAASCH, C.; HERSTATT, C.; LOCK, P. The dynamics of user innovation: drivers and impediments of innovation activities. International Journal of Innovation Management, v. 12, n. 3, p. 377-398, 2008. http://dx.doi.org/10.1142/ S1363919608002060

REICHHELD, F.; MARKEY, R.; OPTON, C. The loyalty effect: the relationship loyalty and profit. European Business Journal, p. 134-139, 2000.

RITTER, T.; WALTER, A. Relationship specific antecedents of customer involvement in new product development. International Journal of Technology Management, v. 26, n. 5-6, p. 482-498, 2003. http://dx.doi.org/10.1504/IJTM.2003.003419

ROWLEY, J.; KUPIEC-TEAHAN, B.; LEEMING, E. Customer community and co-creating: a case study. Marketing Intelligence \& Planning, v. 25, n. 2, p. 136-146, 2007. http:// dx.doi.org/10.1108/02634500710737924

SANDÉN, B. The customer's role in new service development. 2007. 207 f. Tese (Doctor of Management)-Faculty of Economic Science, Communication and IT Business Administration, Karlstad University, Sweden.

SCHREIER, M.; PRUGL, R. Extending lead user theory: antecedents and consequences consumers lead-userness. The Journal of Product Innovation Management, v. 25, p. 331-346, 2008. http://dx.doi.org/10.1111/j.1540-5885.2008.00305.x

SHAH, S. G. S; ROBINSON, 1. Benefits of and barriers to involving user in medical device technology development and evaluation. International Journal of Technology Assessment in Health Care, v. 23, n. 1, p. 131-137, 2007. http://dx.doi. org/10.1108/09526860610687619

SHAH, S. G. S; ROBINSON, 1. User involvement in healthcare technology development and assessment. International Journal of Health Care Quality Assurance, v. 19, n. 6, p. 500-515, 2006. http://dx.doi.org/10.1108/09526860610687619
SIOUKAS, A. V. User involvement for effective customization: an empirical study on voice networks. IEEE Transactions on Engineering Management, v. 42, n. 1, p. 39-49, 1995. http:// dx.doi.org/10.1109/17.366402

SU, C. T.; CHEN, Y; SHA, Y. Linking innovative product development with customer knowledge: a data mining approach. Technovation, v. 26, p. 784-795, 2006. anning, v. 25, n. 2, p. 136-146, 2007. http://dx.doi.org/10.1016/j.technovation.2005.05.005

ULWICK, A. W. Turn customer input into innovation. Harvard Business Review, p. 5-11, 2002.

von HIPPEL, E. Lead Users: a source of novel product concepts. Management Science, v. 32, n. 7, p. 791-805, 1986. http:// dx.doi.org/10.1287/mnsc.32.7.791

von HIPPEL, E. Economics of product development by users: the impact of "Sticky" local information. Management Science, v. 44 , n. 5, 1998.

von HIPPEL, E. Democratizing Innovation. Cambridge: MIT Press, 2005.

von HIPPEL, E. Perspective: user toolkits for innovation. The Journal of Product Innovation Management, v. 18, p. 247-257, 2001. http://dx.doi.org/10.1016/S0737-6782(01)00090-X

von HIPPEL, E.; KATZ, R. Shifting innovation to users via toolkits. Management Science, v. 48, n. 7, 2002. http://dx.doi. org/10.1287/mnsc.48.7.821.2817

von HIPPEL, E.; THOMKE, S.; SONNACK, M. Creating breakthroughs at 3M. Harvard Business Review, v. 77, n. 5, p. 47-57, 1999.

voss, C. et al. Case Research in operations management. International Journal of Operations \& Production Management, v. 22, n. 2. p. 195-219, 2002. http://dx.doi. org/10.1108/01443570210414329

WARNKE, P.; WEBER, M.; LEITNER, K. Transition pathways toward user-centric innovation. International Journal of Innovation Management, v. 12, n. 3, p. 489-510, 2008. http://dx.doi. org/10.1142/S136391960800200X

WEBSTER, J. \& WATSON, R. T. Analyzing the past to prepare for the future: writing a literature review. MIS Quarterly, v. 26, n. 2, p. 13-23, 2002.

YIN, R. K. estudo de caso: planejamento e métodos. 2. ed. Porto Alegre: Bookman, 2001.

\title{
Customer integration in the product development process: a systematic bibliographic review and themes for research
}

\begin{abstract}
This paper presents a systematic bibliographic review and points out research directions for the Customer Integration in the Product Development Process theme. This theme is considered significant in the bibliography of Marketing, Quality, Technological Innovation and Competitive Strategy, because it enables companies to know better the real market needs, contribute to the development of better products, and use the knowledge gained by customers. 72 articles that address this issue, from 1980 to 2008, were collected. The interest in the topic by the academic area has increased significantly since 2002; it can be noted by the growth on the amount of articles published and by the incorporation of this theme in books chapters on management of product development. The following dimensions that involve the subject and predominate in the articles analyzed are identified below: fundamentals for customers' integration, criteria by the choice of customers, types of interaction between the enterprise, and its customers and the benefits obtained by the customers' integration on product development. Future in-depth studies, through case study and research, is recommended on the understanding of the theme, the practices and relations between the dimensions, as well as on the relations of cause and effect between the practices and the performance of product development process.
\end{abstract}

\section{Keywords}

Product development. Customer integration. Systematic bibliographic review. Customer integration on product development. 